\title{
Synthesis of the Impulse Response of the Transmission Path in a Selected Area of the Sound Field
}

\author{
I. CzajkA*, K. Suder-DęBSKa, A. GoŁaś \\ AGH - University of Science and Technology, Department of Power Engineering and Environmental Protection \\ al. Mickiewicza 30, 30-059 Krakow, Poland
}

\begin{abstract}
Providing appropriate sound field parameters in the listening area is very important. It often determines the possibilities of being able to use a facility. Assuming that the sound system is a linear object, it can be described by the impulse response. Unfortunately, it is not possible to designate a single impulse response for such a facility because it is a continuous system. Thus each path between the transmitter and the receiver has its own impulse response. Therefore, the authors have made an attempt to synthesize the impulse response for transmitter-receiver paths with suitable parameters of the sound field in the neighbourhood of the receivers. A technical implementation of the presented synthesis will take place through introduction of additional sources. An experimental determination of the impulse response is relatively simple. The transient response can be numerically determined by using, for example, the finite element method or the boundary element method. Unfortunately, determining the impulse response through simulation of, e.g., planned objects, is much more difficult due to the high computational cost and the lack of precise data on the properties of the materials. For this reason, the authors used an energetic analogue of the impulse response, the echogram.
\end{abstract}

DOI: 10.12693/APhysPolA.123.1072

PACS: 43.55.Ka, 43.20.Dk, 43.40.Rj, 43.55.-n, 43.55.+p, 43.55.Jz, 43.58.Ta

\section{Introduction}

The basic function of a sound reinforcement system is to ensure the required quality of a listening experience. Proper values of parameters describing the sound field are necessary in order to objectively determine that the appropriate design of a sound reinforcement system has been made. Simultaneously, the requirements of the sound system are strongly dependent on the type of broadcast.

Sound systems can be divided into the systems for sound reinforcement in open areas and the systems for sound reinforcement in rooms. Both groups of sound systems are designed to provide the appropriate conditions of listening. These systems have to fulfil the different requirements depending on the type of audio broadcasts. Since the seventies of the last century, more and more sophisticated sound systems are designed and constructed. They enable, among other things, to change of the room acoustic parameters by, for example, apparent lengthening of the reverberation time. Among these systems, SIAP, IOSONO and LARES [1-4] should be mentioned as well as other reverberation enhancement systems [5]. These systems were designed to allow universal use of auditorium halls to reception of different kinds of music and speech broadcasts.

At the end of the 1990s, a lot of work on the sound field reproduction occurred, such as the auralization systems, designed to use for the listening of broadcasts in virtual

*corresponding author; e-mail: iczajka@agh.edu.pl rooms and they are used both for design work and entertainment purposes. Some of them use the image source method, such as [6] or [7].

Despite the technological progress associated with the audio technology and widespread introduction of the digital technology to sound systems, no similar development could be observed in sound reinforcement systems for open areas. One of the most interesting ideas is the Wave Field Synthesis in which reproduction of a sound field is carried out in an anechoic area.

The basic shortage appearing while listening to broadcasts in open areas is the lack of spatial sound experience, associated with the lack of reflections from the surfaces limiting the reinforced area. To eliminate this deficiency, a unique sound system was developed based on the inverse image source method [8-10]. The use of such a system allows for similar listening experience such as those that occur in enclosed spaces. At the same time, electronic implementation of sound rays reflections from the walls allows for optimal selection of the absorption coefficients of the materials used on the walls.

If an acoustic object, understood as the area in which the acoustic wave propagates, is assumed to be linear, it can be described by the impulse response. It is obvious that the acoustic object as a distributed parameter system can be characterised by many impulse responses [11]. Therefore, the notion of the impulse response of the acoustic object will be understood as the impulse response determined between two points - the transmission point and the reception point. The concept of the room impulse response shall be understood as the inverse Fourier transform of the spectral transfer function: 


$$
g(t)=\int_{-\infty}^{\infty} G(f) e^{2 \pi \mathrm{i} f t} \mathrm{~d} f .
$$

A room impulse response, understood in this way, fully characterises the changes in the signal between the transmission and reception points in the sound field. Moreover, on the basis of the impulse response of the transmission path, one can calculate the objective parameters characterising the sound field at the reception point.

Measuring the impulse response of a room is a quite well-known issue and poses no problem [12]. However, providing a synthetic impulse response of designed objects is much more difficult and often exceeds the calculating capabilities of available computers. For that reason, and for further reasoning, an echogram will be used as an easy-to-calculate analogue of the impulse response. The easiest way of obtaining an echogram is to use the geometrical methods of the sound field analysis. An echogram presents the energies of the individual sound corpuscles coming to the receiver in successive moments of time.

Methods to determine the parameters of the sound field based on the echogram have been developed which allow them to be used for the design and analysis of acoustic objects. For these reasons the echogram will be used in further considerations.

Because different types of broadcasts transmitted to listeners have different requirements, often conflicting with one another, it is difficult to build a versatile sound reinforcement system. This system would have to be able to move the speech signal well and provide good quality reception of different types of music. For this reason it seems necessary to modify the properties of the sound field at the reception point. Such modifications can be made by modifying the impulse response or the echogram.

The authors participated in creating a sound system based on the inverse image source method [8]. This sound system allows to obtain an acoustic field in the open space with parameters that are similar to the parameters of the sound field in enclosures [13]. Simplifying, it can be said that the system is done according to the following algorithm [8, 10]:

- generating a grid of image sources until it reaches the selected row for a given geometry of the reinforcement area;

- finding intersection points of the area's border by rays from the image sources to the receivers;

- determining the position of the real sources based on the concentration of intersection points;

- determining the delay and gain coefficients for signals corresponding to individual image sources;

- implementing filter banks of selected reinforcements and delays in each channel.
As has been demonstrated earlier [9], the sound system allows to obtain, inter alia, spatial sound impressions in open areas.

\section{Posing the problem}

The main task is to modify the impulse response (or the echogram) for selected paths from the sound source to the receiver to achieve the required values of parameters characterising the sound field. The following parameters have been chosen for describing the sound field at the reception point [14]:

- definition $D_{50}$

$$
D_{50}=\frac{\int_{0}^{50 \mathrm{~ms}} p^{2}(t) \mathrm{d} t}{\int_{0}^{\infty} p^{2}(t) \mathrm{d} t} \cdot 100 \%,
$$

- clarity $C_{80}$

$$
C_{80}=10 \log \frac{\int_{0}^{80 \mathrm{~ms}} p^{2}(t) \mathrm{d} t}{\int_{80 \mathrm{~ms}}^{\infty} p^{2}(t) \mathrm{d} t},
$$

- early lateral fraction

$$
L F_{0}^{80}=\frac{\int_{5 \mathrm{~ms}}^{80 \mathrm{~ms}} p_{\infty}^{2}(t) \mathrm{d} t}{\int_{0}^{80 \mathrm{~ms}} p^{2}(t) \mathrm{d} t} \cdot 100 \%
$$

In all of the above definitions, $p(t)$ is the sound pressure measured by the transducer of the omnidirectional directivity pattern, while $p_{\infty}(t)$ is the sound pressure measured by the transducer of the figure-of-eight directivity pattern.

Because the selected parameters allow to estimate the quality of a speech signal and music signal of varying nature, in the opinion of the authors they are universal, and at the same time they do not require complex calculations. This is particularly important if the plan is to obtain implementation of this method in real sound systems must be mobile by assumption.

Depending on the type of broadcast, the recommended value of the parameter should be slightly different. And so, in order to ensure good speech intelligibility it is necessary that the sound field parameters take the following values: $C_{50}>2 \mathrm{~dB} ; S T I>0.6 ; D_{50}>70 \%$; and $\% A L_{\text {cons }}<10 \%$ [15]. On the other hand, the following parameter values are recommended for music: $C_{80}>-2$ $\mathrm{dB}$ close to the sound source; $C_{80}>-5 \mathrm{~dB}$ far from the sound source; $L F_{0}^{80}=15 \%-25 \%$ [15].

An echogram is a graphical representation of the energy reaching the receiver in successive moments of time. Geometric methods use sound corpuscles that carry portions of energy from the sound source to the receiver; these are subject to the laws of geometric reflection and thus reduce their energy according to the sound absorption coefficient of the walls from which they are reflected. The subsequent sound corpuscles reaching the receiving point are recorded as the subsequent stripes of an echogram (Fig. 1). 


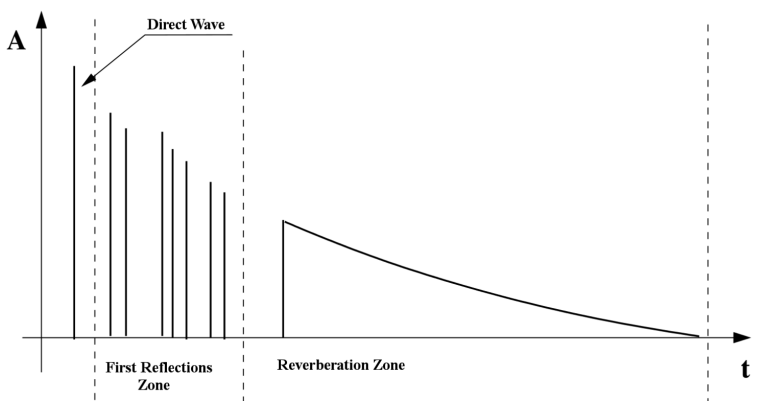

Fig. 1. Example of an echogram with its characteristic parts.

In this way an echogram characterises the temporal variations of acoustic energy in the analysed point of the sound field. Thus some characteristic elements, marked in Fig. 1, can be distinguished in the echogram.

The analysed area, with dimensions of $12 \times 20$ meters, is shown in Fig. 2. "Virtual" walls are situated around the reinforced area and are then mapped by the sound system through adequate signal modifications [10]. In the

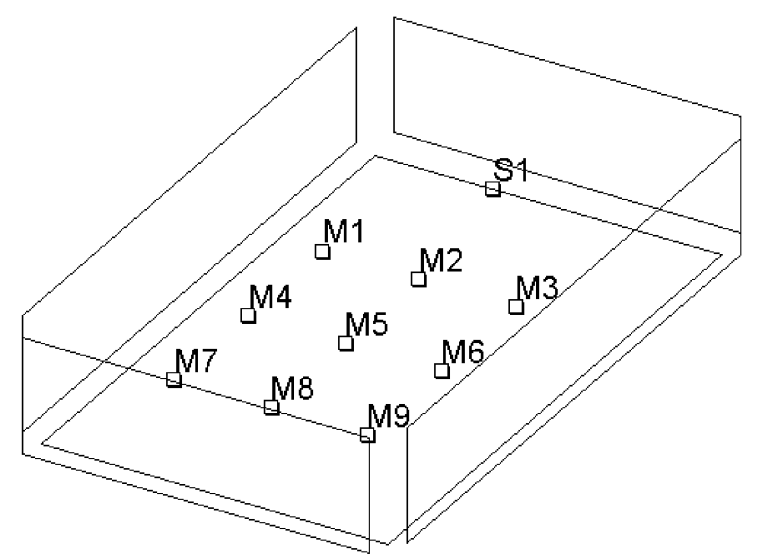

Fig. 2. Analysed area with source (S) and receivers $(\mathrm{M})$.

basic version, i.e. without modification of an echogram, the following values of the surface sound absorption coefficients have been adopted: for ground, $\alpha=0.04$, and for walls, $\alpha=0.1$.

Respectively, by modifying the sound signal, the required characteristics of the signal at the receiving point may be obtained. In a further part of the considerations, two methods of modifying the signal emitted by the Inverse Sound System for Open Areas will be presented.

\section{Modification of an impulse response}

By using the described method, modifications of an impulse response can be carried out in two ways. The first is the introduction of a time-varying sound absorption coefficient of the virtual walls of the modelled room. Another way consist in introducing additional virtual reflective "planes" (Fig. 3).

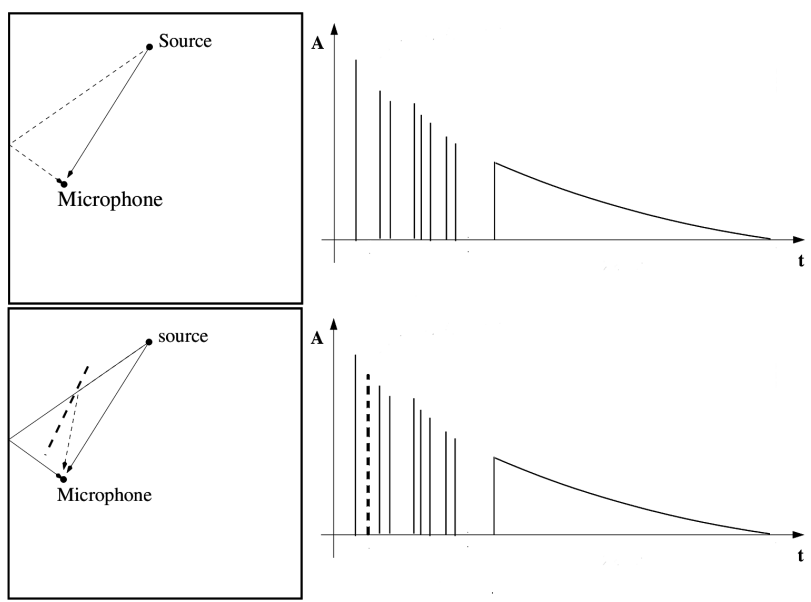

Fig. 3. Concept of generating additional stripes in the echogram.

Modifying the geometry of the virtual room leads to appearance of additional stripes in arbitrarily selected points of the echogram. It is important to realise that this additional reflecting surface also causes appearance of additional stripes in the later part of the echogram.

For that reason it is preferred to introduce a timevarying sound absorption coefficient. Such a procedure allows to determine the height of individual stripes.

A combination of both these methods is the most beneficial because it lets us to add stripes to the originalimportant due to the described parameters of the signal transmitted in the sound field-and prevents the addition of energy to the so-called reverberant tail.

\section{Results of simulation studies}

The following figures show some of the results of numerical analyses. For each of the results on the left, changes of the sound absorption coefficient of virtual walls at time are shown, of which the middle includes tables of the values of the parameters at all points of measurement, while on the right there is an echogram for measuring point number 5 . A total of 10 reflections have been taken into account for all of the analysed cases. The calculations were made using the image source method. 
TABLE

Parameters of the sound field:

\begin{tabular}{|c|c|c|c|c|c|c|c|c|c|}
\hline No. & 1 & 2 & 3 & 4 & 5 & 6 & 7 & 8 & 9 \\
\hline \multicolumn{10}{|c|}{ without impuls response modification } \\
\hline$C_{80}$ & 10.533 & 12.657 & 10.532 & 6.8618 & 8.2193 & 6.8589 & 4.0274 & 4.4847 & 4.0275 \\
\hline$D_{50}$ & 85.73 & 88.918 & 85.728 & 64.597 & 76.901 & 64.59 & 48.989 & 44.419 & 48.989 \\
\hline$L F_{0}^{80}$ & 22.078 & 15.812 & 22.078 & 44.454 & 15.231 & 44.454 & 40.913 & 21.767 & 40.913 \\
\hline \multicolumn{10}{|c|}{ with a linear change of $\alpha$ from 1 to 0 at $300 \mathrm{~ms}$} \\
\hline$C_{80}$ & 13.735 & 16.845 & 13.735 & 9.0764 & 12.16 & 9.0711 & 5.5885 & 8.0562 & 5.5878 \\
\hline$D_{50}$ & 95.555 & 97.699 & 95.555 & 85.638 & 93.007 & 85.627 & 71.225 & 77.92 & 71.222 \\
\hline$L F_{0}^{80}$ & 3.982 & 7.1405 & 3.982 & 12.947 & 6.7151 & 12.947 & 15.122 & 8.9889 & 15.122 \\
\hline \multicolumn{10}{|c|}{ with a linear change of $\alpha$ from 0 to 1 at $300 \mathrm{~ms}$} \\
\hline$C_{80}$ & 16.091 & 17.377 & 16.091 & 12.446 & 14.265 & 12.447 & 9.5589 & 9.8969 & 9.5589 \\
\hline$D_{50}$ & 92.687 & 93.091 & 92.687 & 76.926 & 87.51 & 76.926 & 65.289 & 57.942 & 65.289 \\
\hline$L F_{0}^{80}$ & 20.896 & 14.952 & 20.896 & 42.789 & 13.968 & 42.789 & 39.679 & 20.132 & 39.679 \\
\hline \multicolumn{10}{|c|}{ with a linear change of $\alpha$ from 1 to 0 at $100 \mathrm{~ms}$} \\
\hline$C_{80}$ & 7.8789 & 10.728 & 7.8788 & 3.5896 & 5.821 & 3.5895 & 0.75299 & 2.0989 & 0.75288 \\
\hline$D_{50}$ & 82.625 & 89.844 & 82.625 & 58.547 & 73.302 & 58.547 & 39.357 & 43.985 & 39.357 \\
\hline$L F_{0}^{80}$ & 11.231 & 10.414 & 11.231 & 29.05 & 11.171 & 29.05 & 31.329 & 16.911 & 31.329 \\
\hline \multicolumn{10}{|c|}{ with a linear change of $\alpha$ from 0 to 1 at $100 \mathrm{~ms}$} \\
\hline$C_{80}$ & 28.08 & 27.494 & 28.08 & 27.551 & 36.742 & 27.551 & 25.906 & 25.948 & 25.906 \\
\hline$D_{50}$ & 98.726 & 98.066 & 98.726 & 92.073 & 97.457 & 92.073 & 86.903 & 79.095 & 86.903 \\
\hline$L F_{0}^{80}$ & 15.122 & 11.236 & 15.122 & 33.277 & 9.6303 & 33.277 & 31.419 & 13.814 & 31.419 \\
\hline \multicolumn{10}{|c|}{ with a linear change of $\alpha$ from 1 to 0 at $200 \mathrm{~ms}$} \\
\hline$C_{80}$ & 10.571 & 13.577 & 10.571 & 5.8842 & 8.6815 & 5.8855 & 2.5264 & 4.6798 & 2.5261 \\
\hline$D_{50}$ & 91.087 & 95.188 & 91.086 & 74.458 & 85.865 & 74.462 & 55.194 & 63.53 & 55.193 \\
\hline$L F_{0}^{80}$ & 5.6413 & 7.8172 & 5.6413 & 17.162 & 7.64 & 17.162 & 20.161 & 10.944 & 20.161 \\
\hline \multicolumn{10}{|c|}{ with a linear change of $\alpha$ from 0 to 1 at $200 \mathrm{~ms}$} \\
\hline$C_{80}$ & 18.822 & 19.524 & 18.822 & 15.3 & 17.75 & 15.3 & 12.517 & 12.907 & 12.517 \\
\hline$D_{50}$ & 94.99 & 94.77 & 94.99 & 81.842 & 91.196 & 81.842 & 72.057 & 64.275 & 72.057 \\
\hline$L F_{0}^{80}$ & 19.332 & 13.919 & 19.332 & 40.512 & 12.756 & 40.512 & 37.961 & 18.531 & 37.961 \\
\hline \multicolumn{10}{|c|}{ with a step change of $\alpha$ from 0 to 1 at $90 \mathrm{~ms}$} \\
\hline$C_{80}$ & 17.228 & 16.342 & 17.228 & 13.986 & 15.301 & 13.986 & 10.918 & 11.746 & 10.918 \\
\hline$D_{50}$ & 90.168 & 90.311 & 90.168 & 72.302 & 84.093 & 72.302 & 60.888 & 53.406 & 60.888 \\
\hline$L F_{0}^{80}$ & 24.263 & 17.204 & 24.263 & 47.116 & 16.535 & 47.116 & 42.537 & 23.256 & 42.537 \\
\hline \multicolumn{10}{|c|}{ with a step change of $\alpha$ from 1 to 0 at $90 \mathrm{~ms}$} \\
\hline$C_{80}$ & 7.5795 & 11.693 & 7.5794 & 2.17 & 5.3301 & 2.1699 & -1.9522 & 0.73897 & -1.9523 \\
\hline$D_{50}$ & 85.135 & 93.658 & 85.135 & 62.238 & 77.335 & 62.237 & 38.948 & 54.244 & 38.947 \\
\hline$L F_{0}^{80}$ & 0.90991 & 6.0742 & 0.90991 & 4.2887 & 5.3892 & 4.2887 & 2.6292 & 5.392 & 2.6292 \\
\hline \multicolumn{10}{|c|}{ with a step change of $\alpha$ from 0 to 1 at $110 \mathrm{~ms}$} \\
\hline$C_{80}$ & 13.41 & 15.687 & 13.41 & 9.9935 & 11.019 & 9.9935 & 7.117 & 7.3818 & 7.117 \\
\hline$D_{50}$ & 87.868 & 89.979 & 87.868 & 68.345 & 80.229 & 68.345 & 55.112 & 48.175 & 55.112 \\
\hline$L F_{0}^{80}$ & 24.263 & 17.204 & 24.263 & 47.116 & 16.535 & 47.116 & 42.537 & 23.256 & 42.537 \\
\hline \multicolumn{10}{|c|}{ with a step change of $\alpha$ from 1 to 0 at $110 \mathrm{~ms}$} \\
\hline$C_{80}$ & 8.7176 & 11.99 & 8.7175 & 3.2891 & 6.5518 & 3.289 & -0.82134 & 1.9789 & -0.82149 \\
\hline$D_{50}$ & 88.156 & 94.053 & 88.156 & 68.078 & 81.885 & 68.077 & 45.286 & 61.198 & 45.285 \\
\hline$L F_{0}^{80}$ & 0.90991 & 6.0742 & 0.90991 & 4.2887 & 5.3892 & 4.2887 & 2.6292 & 5.392 & 2.6292 \\
\hline \multicolumn{10}{|c|}{ with a step change of $\alpha$ from 0 to 1 at $130 \mathrm{~ms}$} \\
\hline$C_{80}$ & 12.101 & 14.026 & 12.101 & 8.3603 & 9.3123 & 8.3603 & 5.4544 & 5.7707 & 5.4544 \\
\hline$D_{50}$ & 86.54 & 88.891 & 86.54 & 65.618 & 77.495 & 65.618 & 51.226 & 45.049 & 51.226 \\
\hline$L F_{0}^{80}$ & 24.263 & 17.204 & 24.263 & 47.116 & 16.535 & 47.116 & 42.537 & 23.256 & 42.537 \\
\hline \multicolumn{10}{|c|}{ with a step change of $\alpha$ from 1 to 0 at $130 \mathrm{~ms}$} \\
\hline$C_{80}$ & 9.5808 & 13.157 & 9.5807 & 4.3861 & 7.7977 & 4.3859 & 0.35821 & 3.1371 & 0.35801 \\
\hline$D_{50}$ & 90.079 & 95.389 & 90.079 & 73.301 & 85.76 & 73.3 & 52.061 & 67.312 & 52.06 \\
\hline$L F_{0}^{80}$ & 0.90991 & 6.0742 & 0.90991 & 4.2887 & 5.3892 & 4.2887 & 2.6292 & 5.392 & 2.6292 \\
\hline
\end{tabular}




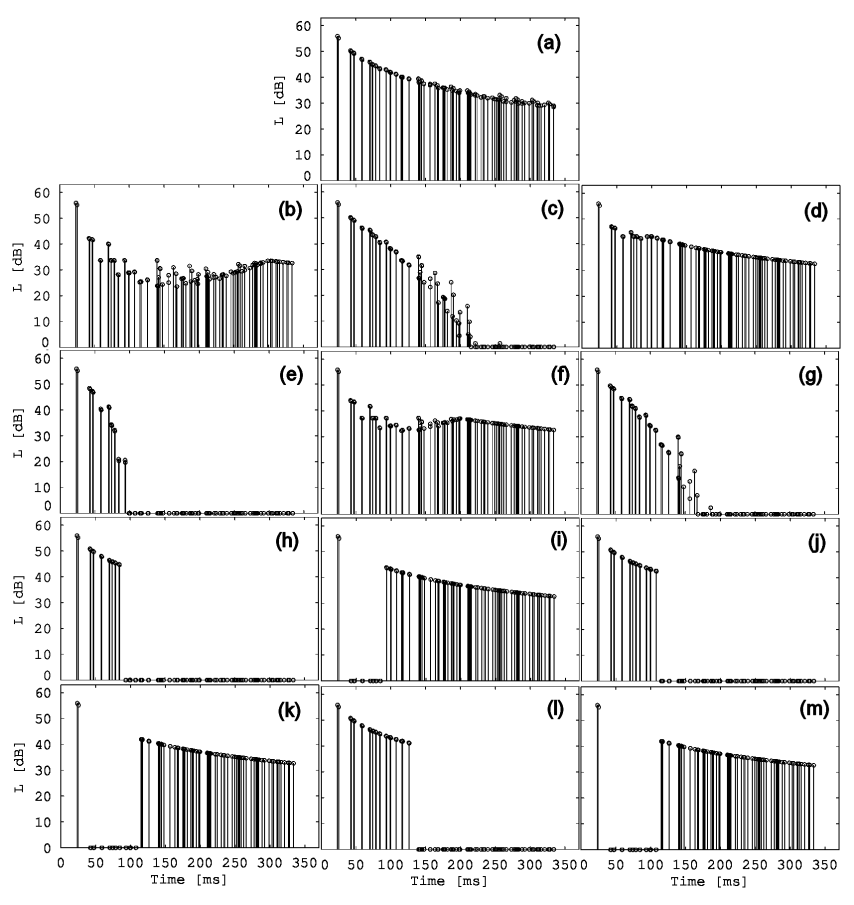

Fig. 4. Echogram at point 5: (a) without impuls response modification and, (b) with a linear change of $\alpha$ from 1 to 0 at $300 \mathrm{~ms}$, (c) with a linear change of $\alpha$ from 0 to 1 at $300 \mathrm{~ms}$, (d) with a linear change of $\alpha$ from 1 to 0 at $100 \mathrm{~ms}$, (e) with a linear change of $\alpha$ from 0 to 1 at $100 \mathrm{~ms}$, (f) with a linear change of $\alpha$ from 1 to 0 at $200 \mathrm{~ms}$, (g) with a linear change of $\alpha$ from 0 to 1 at $200 \mathrm{~ms}$, (h) with a step change of $\alpha$ from 0 to 1 at $90 \mathrm{~ms}$, (i) with a step change of $\alpha$ from 1 to 0 at $90 \mathrm{~ms}$, (j) with a step change of $\alpha$ from 0 to 1 at $110 \mathrm{~ms}$, (k) with a step change of $\alpha$ from 1 to 0 at $110 \mathrm{~ms}$, (1) with a step change of $\alpha$ from 0 to 1 at $130 \mathrm{~ms},(\mathrm{~m})$ with a step change of $\alpha$ from 1 to 0 at $130 \mathrm{~ms}$.

Figure 4a presents simulation results for the primary area: the absorption coefficients of walls, $\alpha=0.1$, and the absorption coefficient of the floor, $\alpha=0.04$.

The values of the coefficients shown in Fig. 4a indicate quite poor quality of speech and music reception in the analysed area. The worst conditions of listening experience prevail in the last row which is the most distant from the sound source.

Figure $4 \mathrm{~b}$ presents the results for the walls with a linear change of the sound absorption coefficient $\alpha$ from 1 to 0 at $300 \mathrm{~ms}$.

The values of the coefficients indicate a good speech intelligibility, while the conditions of the listening experience of music are not too good. The sound is too dryespecially at the front, with a lack of spatial impressions.

Figure $4 \mathrm{c}$ presents the results for the walls with a linear change of the sound absorption coefficient $\alpha$ from 0 to 1 at $300 \mathrm{~ms}$. The system is not suitable for listening to speech and music. In the case of music the sound is too dry and selective with spatial impressions that are too large. For these reason, poor speech intelligibility is in the last row.
The results for the walls with a linear change of sound absorption coefficient $\alpha$ from 1 to 0 at $100 \mathrm{~ms}$ are shown in Fig. 4 d. The values of the $L F_{0}^{80}$ coefficient at all points are close to the recommended ones.

There are good conditions for a listening experience of different genres of music. Speech intelligibility is not too good. This case can be considered as good for pre-tuning the system to reinforce the music.

The results for the walls with a linear change of sound absorption coefficient $\alpha$ from 0 to 1 at $100 \mathrm{~ms}$ are shown in Fig. 4e. With such characteristics of the sound absorption coefficients, the system reflects speech very well. However, music will be deprived of appropriate aesthetic qualities. This case can be considered as recommended for the transmission of an exclusively verbal broadcast.

Figure $4 \mathrm{f}$ shows the results of calculations when a linear change of the sound absorption coefficient from 1 to 0 takes place at $200 \mathrm{~ms}$. They indicate good quality reception of both speech and music, even though there are worse conditions for the listening experience of speech in the last row, and in the first row the reception of music is worse; however, this case can be considered to be the system's universal set of the system for transmitting broadcasts with elements of both speech and music. One can see a significant improvement as compared to the basic version without a modification of the sound absorption coefficient of the walls.

The results of the linear change of the sound absorption coefficient from 0 to 1 at $200 \mathrm{~ms}$ are shown in Fig. $4 \mathrm{~g}$. A speech intelligibility improvement is clearly noticeable but it is not as large as in the case shown in Fig. 4e. However, when transmitting music, the sound is very selective and dry.

Below are the results for a step change of the sound absorption coefficient occurring in an arbitrary time moment at switching times less than $90 \mathrm{~ms}$. It was not possible to determine the value of $C_{80}$, therefore, there have been presented the results for the moments of switching from $90 \mathrm{~ms}$ to $130 \mathrm{~ms}$. Figure $4 \mathrm{~h}$ shows the values of parameters characterising the sound field at the step change in the sound absorption coefficient between 0 and 1 at $90 \mathrm{~ms}$. As one can see, the result is a very short echogram, with very strong influence of lateral reflections, not very good speech intelligibility, and music without a large aesthetic value.

When the direction of the changes in the value of the sound absorption coefficient for a change from 1 to 0 at $90 \mathrm{~ms}$ is altered, one obtains results as those shown in Fig. 4i. The values of coefficients $L F_{0}^{80}, C_{80}$ and $D_{50}$ indicate poor speech intelligibility; even though almost all of $C_{80}$ values are within the prescribed limits, values of the $L F_{0}^{80}$ parameter indicate the fact that spatial impressions will be missing at the perception of music.

The next two cases show the step change of the sound absorption coefficient at $110 \mathrm{~ms}$. Figure $4 \mathrm{j}$ presents a change from 0 to 1 . The results are very similar to those obtained with the same change at $90 \mathrm{~ms}$. One can see a slight improvement in the reception of music. 
For a step change of the sound absorption coefficient from 1 to 0 , at $110 \mathrm{~ms}$ the value of coefficients characterising a sound field, as shown in Fig. 4k, can be obtained. Speech intelligibility is still not satisfactory and perception of music is better than before, although there is a lack of spatial impressions.

When a step change of the sound absorption coefficient from 0 to 1 occurs at $130 \mathrm{~ms}$, values of parameters as shown in Fig. 41 are obtained. These indicate not very good conditions of listening experience both for speech and music.

For a change of the sound absorption coefficient from 0 to 1 , values of parameters as shown in Fig. $4 \mathrm{~m}$ are obtained.

Poor speech intelligibility occurs in back rows and there is good selectivity of music, but there is a lack of spatial impressions. Therefore, this case should also be considered as not very useful for practical applications.

\section{Summary and conclusions}

The sound field generated by a system based on the inverse image source method can be created for a very wide range of applications. These modifications can be described using the impulse responses of paths between the source and the receiver. In the acoustics of rooms, the echogram is the easiest way to obtain an equivalent of the impulse response.

The paper presents two ways of modify the impulse response of sound transmission paths in a selected area of the sound field. The first one concerns the introduction of a variable sound absorption coefficient of virtual walls of a room, while the other comes down to the modifying the geometry of virtual walls which leads to a change the layout of the strips in the echogram.

The first way is discussed in detail, giving the examples of characteristics of sound absorption coefficients.

The results show that the described way of modifying the signal is a tool which enables to modify the parameters of the sound field in a wide range. This allows to adapt the system to the nature of the transmitted signal.

The paper presents two methods of modifying the impulse response of the transmission of sound in a selected area of the sound field. The first way concerns the introduction of a variable sound absorption coefficient of the virtual walls of a room, while the other is basically a modification of geometry of virtual walls which leads to a change in the layout of stripes in the echogram.

The studies presented here are of a numerical character, and an experimental verification of the presented results seems to be necessary. Because the modifications of the signal transmitted by each sound source are made with the help of filters, modifications of the signal phase are made in addition to the amplitude changes. For that reason it is necessary to examine whether implementation of these methods does not introduce phase distortions that significantly degrade the listening experience.

The described method can be used not only to reinforce the open area but also to improve the acoustics of existing rooms. This problem becomes much more difficult to solve then.

The described method of modifying the impulse response of the transmission path in a selected area of the sound field can be used to create the eligible sound experience in open areas. Owing to the fact, it is possible to apply the described sound reinforcement system to various types of the broadcasts in order to obtain the best possible aesthetic experience in the open area.

\section{References}

[1] B. van Munster, W. Prinssen, Proc. 146th Meeting: Acoust. Soc. Am. 2003.

[2] S. Mauer, F. Melchior, Proc. 130th AES Conv., London 2011.

[3] F. Melchior, J. Ahrens, S. Spors, Proc. 129th AES Conv., San Francisco 2010.

[4] D. Griesinger, Proc. 90th AES Conv., Paris 1991.

[5] A. C. Grade, Acta Acust. 83, 522 (1997).

[6] M. Noisternig, B. F. G. Katz, S. Siltanen, L. Savioja, Acta Acust. 94, 1000 (2008).

[7] S. Favrot, J. M. Buchholz, Acta Acust. 96, 364 (2010).

[8] A. Gołaś, K. Suder-Dębska, Archiv. Acust. 37, 323 (2012).

[9] A. Gołaś, K. Suder-Dębska, W. Ciesielka, R. Filipek, Acta Phys. Pol. A 119, 966 (2011).

[10] A. Gołaś, K. Suder-Dębska, Proc. 17th International Congress on Sound \& Vibration, Cairo 2010.

[11] H. Kuttruff, Room Acoustics, Spon Press, Abingdon 2009.

[12] J.P. Paulo, C.R. Martins, J.L.B. Coelho, Appl. Acoust. 70, 556 (2009).

[13] A. Gołaś, K. Suder-Dębska, Proc. 58th Open Seminar on Acoustics joined with 2nd Polish-German structured conference on Acoustics, Gdańsk-Jurata, Poland 2011.

[14] F. P. Mechel, Formulas of Acoustics, SpringerVerlag, Berlin-Heidelberg-New York 2008.

[15] A. Kulowski, Room Acoustics: design recommendations for architects, Wyd. Politechniki Gdańskiej, Gdańsk 2011, [in Polish]. 